\title{
BMJ Open Clinician perceptions of a prototype wearable exercise biofeedback system for orthopaedic rehabilitation: a qualitative exploration
}

\author{
Rob Argent, ${ }^{1,2,3}$ Patrick Slevin, ${ }^{2,3}$ Antonio Bevilacqua, ${ }^{2}$ Maurice Neligan, ${ }^{1}$ \\ Ailish Daly, ${ }^{1}$ Brian Caulfield ${ }^{2,3}$
}

To cite: Argent R, Slevin $P$, Bevilacqua A, et al. Clinician perceptions of a prototype wearable exercise biofeedback system for orthopaedic rehabilitation: a qualitative exploration. BMJ Open 2018;8:e026326. doi:10.1136/ bmjopen-2018-026326

- Prepublication history and additional material for this paper are available online. To view these files, please visit the journal online (http://dx.doi org/10.1136/bmjopen-2018026326).

Received 28 August 2018 Revised 2 October 2018 Accepted 2 October 2018
A) Check for updates

(C) Author(s) (or their employer(s)) 2018. Re-use permitted under CC BY-NC. No commercial re-use. See rights and permissions. Published by BMJ.

${ }^{1}$ Beacon Hospital, Dublin, Ireland ${ }^{2}$ Insight Centre for Data Analytics, University College Dublin, Dublin, Ireland

${ }^{3}$ School of Public Health, Physiotherapy and Sport Science, University College Dublin, Dublin, Ireland

Correspondence to

Rob Argent;

rob.argent@insight-centre.org

\section{ABSTRACT}

Objectives This study explores the opinions of orthopaedic healthcare professionals regarding the opportunities and challenges of using wearable technology in rehabilitation. It continues to assess the perceived impact of an exemplar exercise biofeedback system that incorporates wearable sensing, involving the clinician in the user-centred design process, a valuable step in ensuring ease of implementation, sustained engagement and clinical relevance.

Design This is a qualitative study consisting of one-to-one semi-structured interviews, including a demonstration of a prototype wearable exercise biofeedback system. Interviews were audio-recorded and transcribed, with thematic analysis conducted of all transcripts.

Setting The study was conducted in the orthopaedic department of an acute private hospital.

Participants Ten clinicians from a multidisciplinary team of healthcare professionals involved in the orthopaedic rehabilitation pathway participated in the study.

Results Participants reported that there is currently a challenge in gathering timely and objective data for the monitoring of patients in orthopaedic rehabilitation. While there are challenges in ensuring reliability and engagement of biofeedback systems, clinicians perceive significant value in the use of wearable biofeedback systems such as the exemplar demonstrated for use following total knee replacement.

Conclusions Clinicians see an opportunity for wearable technology to continuously track data in real-time, and feel that feedback provided to users regarding exercise technique and adherence can further support the patient at home, although there are clear design and implementation challenges relating to ensuring technical accuracy and tailoring rehabilitation to the individual. There was perceived value in the prototype system demonstrated to participants which supports the ongoing development of such exercise biofeedback platforms.

\section{INTRODUCTION}

It has been estimated that over $10 \%$ of the population will need a total knee replacement (TKR) by 80 years of age. ${ }^{1}$ Home-based exercises following TKR form the mainstay
Strengths and limitations of this study

- There is a need for innovative solutions to counteract poor adherence and increasing pressure on patient self-management, and a user-centred design approach with key stakeholders is recommended.

- Few studies have investigated clinicians' perceptions of exercise biofeedback systems and offered the opportunity for healthcare professionals to contribute to the user-centred design process.

- This study uses qualitative methods in the form of semi-structured interviews to allow for an indepth exploration of participant opinions, yet results are of a subjective nature and are not necessarily generalisable.

- Study participants were recruited from a number of different disciplines across nursing and therapies, although from a single healthcare institution.

of rehabilitation, with typical physiotherapy programmes consisting of exercises targeted to increase strength, range of movement and function as well as reduce postoperative complications. ${ }^{2}$ However, adherence rates to home-based exercises are alarmingly low, with patients reporting various reasons for non-compliance with the prescribed programme. ${ }^{3} 4$ Patients often report a lack of confidence following discharge from hospital, insecurity surrounding their postoperative expectations and poor recall of exercise technique. ${ }^{5}$ Poor performance of exercise technique such as insufficient range of motion, alignment or compensatory movements may impact on the efficacy of the rehabilitation programme, and hence the outcome of the procedure. ${ }^{6}$ Poor adherence may even lead to further complications, readmission to hospital, additional healthcare costs and prolonged pain and disability for the patient. ${ }^{7}$

The lack of support reported by patients may also result from the changing nature 
of joint replacement surgery globally, moving towards value-based care and a drive for reduction in the length of hospital stay, placing a greater emphasis than ever on the self-management skills of the patient. Healthcare providers need to make the most of the opportunity that new and emerging technologies present, ${ }^{18}$ and connected health solutions may provide the opportunity to bridge this gap between the home and clinician. ${ }^{3}$ With the advancement of sensor technologies and mobile computing platforms, it is now possible to empower patients to self-manage more effectively, acquire data with far greater efficiency and use this to support the clinician in making more objective, data-driven decisions about clinical care. ${ }^{8}$ Inertial measurement units (IMUs) can be used to measure the three-dimensional position of a limb segment and have been shown to be a cost-effective, accurate method of assessing exercise technique in rehabilitation exercises, ${ }^{9-12}$ presenting the opportunity to build such a classification system into a connected health intervention.

When designing a connected health intervention, in order to promote user-engagement and maximise impact, an iterative design process involving consultation with key stakeholders is recommended throughout the design and development phase to optimise the effectiveness of the system. ${ }^{1314}$ As well as the patient end-user, clinicians should be encouraged to participate in the design and evaluation of connected health solutions to ensure clinical acceptability. ${ }^{15}$ However, there is currently a lack of collaboration between technology developers and healthcare professionals, which in turn is affecting acceptance and adoption of new technologies. ${ }^{15} 16$

The aim of this study was twofold. In the first instance, we sought to perform an exploration of the opportunities and challenges of using wearable technology in rehabilitation after joint replacement surgery. Following this, clinicians were provided with a demonstration of an exemplar wearable exercise biofeedback system, and their perceptions of its potential use in the clinical setting were evaluated while incorporating the healthcare professional in the user-centred design process.

\section{METHODS}

\section{Patient and public involvement}

No patients or members of the general public were involved in this study.

\section{Participants}

A total of 10 participants (6 females, 4 males, age: 36.5 years (SD 9.06)) from a variety of clinical disciplines (4 physiotherapists, 2 clinical nurse specialists, 2 orthopaedic assistants, 1 occupational therapist and 1 staff nurse) were recruited as a sample of convenience from a private hospital in Dublin, Ireland, and were involved in the care of patients in the orthopaedic joint replacement pathway within the hospital. Potential participants were identified and introduced to the study by an Orthopaedic
Consultant (MN) and the Allied Therapies Manager $(\mathrm{AD})$. Each participant signed a consent form prior to commencing the study.

\section{Experimental procedure}

Participants were required to attend a single hour-long session as part of the study. All interview data were recorded using a Dictaphone and an interview topic guide (see online supplementary file 1) was constructed based on the main research questions and aims of the study, in order to ensure consistency between interviews. ${ }^{17}$ Prior to introducing the concept and exemplar biofeedback system, and to prevent bias, a semi-structured interview was conducted with each participant to gain an understanding of what methods clinicians are currently using to monitor and provide feedback to patients, the perceived opportunities and challenges of connected health interventions, and their current knowledge of wearable technology. A demonstration of the exemplar wearable exercise biofeedback system was then provided, followed by a further semi-structured interview to identify the perceived impact and clinical implications of such a system.

\section{Prototype exercise biofeedback system}

A prototype has been developed consisting of a single IMU (Shimmer, Dublin, Ireland) and an Android application developed for a tablet computer to be used by the patient in their own home. The IMU is placed on the shank in a neoprene sleeve and is connected via Bluetooth to the Android application on the tablet. As the user exercises, the IMU streams sensor data to the tablet, and an on-screen avatar mirrors the movements and the repetitions are counted for each exercise. At the end of the set, the user is provided with feedback on their technique, ${ }^{11} 18$ and if erroneous, advice on how to improve technique is displayed. Patient-reported outcomes are captured on a regular basis and the user's progress is also presented graphically to track adherence. An illustration of the user setup is included in figure 1 with screenshot in figure 2.

\section{Data analysis}

Audio from interview recordings was transcribed and anonymised. Thematic analysis of the interview transcript took place with a grounded-theory approach. ${ }^{17}$ The interview guide was used to create an early coding template, which was then refined and finalised as further themes emerged during data analysis, ${ }^{19}$ conducted by RA (research physiotherapist) and PS (experienced qualitative researcher). A constant comparison approach was taken with regular cross-checking to ensure reliability of emerging subthemes, with a strong correlation between researchers. ${ }^{20}$ Any outliers were discussed, and agreement reached, with data saturation agreed when no further themes were occurring in the interview data. ${ }^{17}$ 


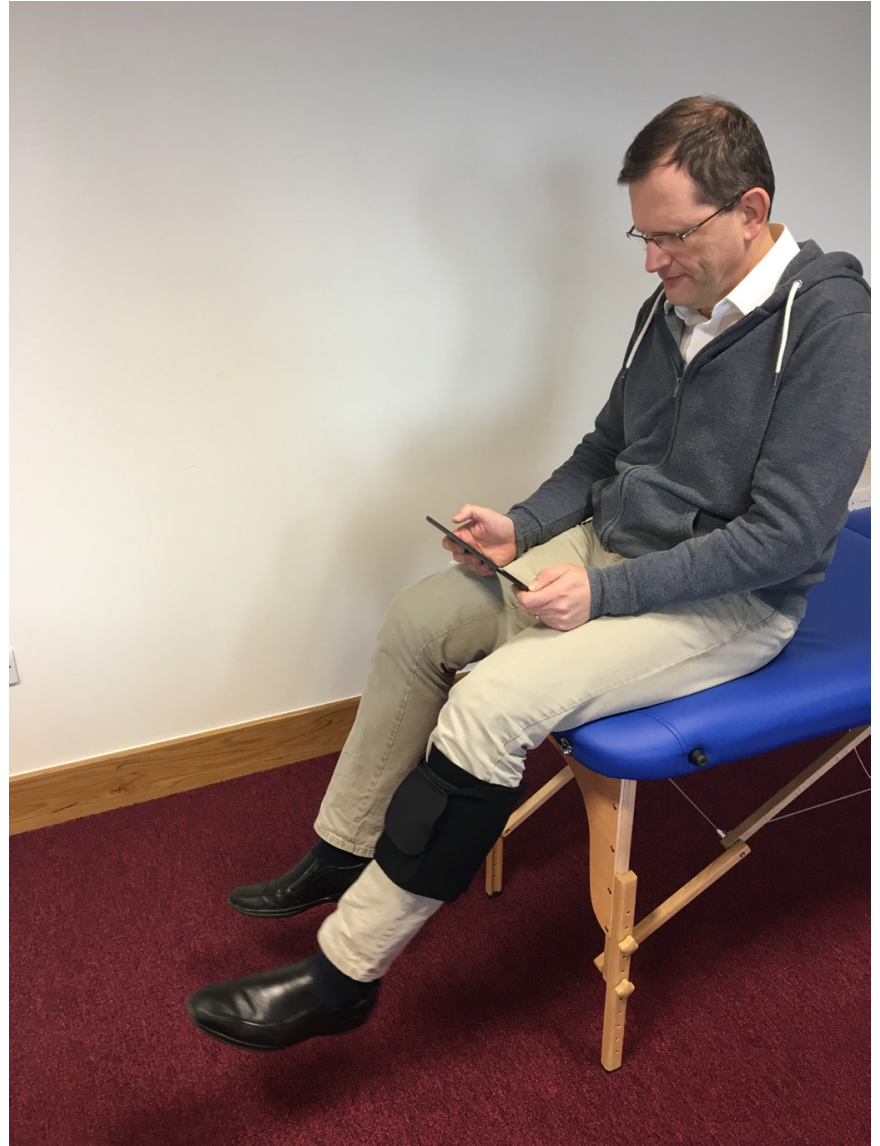

Figure 1 User setup of biofeedback system with single inertial measurement unit placed on the shin and associated tablet application (written consent provided for use of image).

\section{RESULTS}

A summary of results are reported below, additional quotations to support these results can be found in online supplementary file 2.

\section{Current methods of monitoring and feedback}

Clinical practice at present tends to rely on a combination of objective and subjective markers. Participants spoke about the wide use of range of motion measurement as a key objective marker following TKR. Additionally, markers such as swelling, pain and functional scores are used to monitor changes in the patient's condition.

Generally, I would manually take objective measures, traditional measures such as muscle strength, range of motion, and then some subjective ones as well, opinion based on movement quality. (Physiotherapist)

However, participants commented on the lack of objectivity of assessing muscle strength, gait, and exercise technique, with visual assessment and patient self-report forming the basis for ongoing monitoring.

Objective markers are few and far between and we're still reliant on the old clinical measures like range of motion, muscle strength-which if you're
Exercises
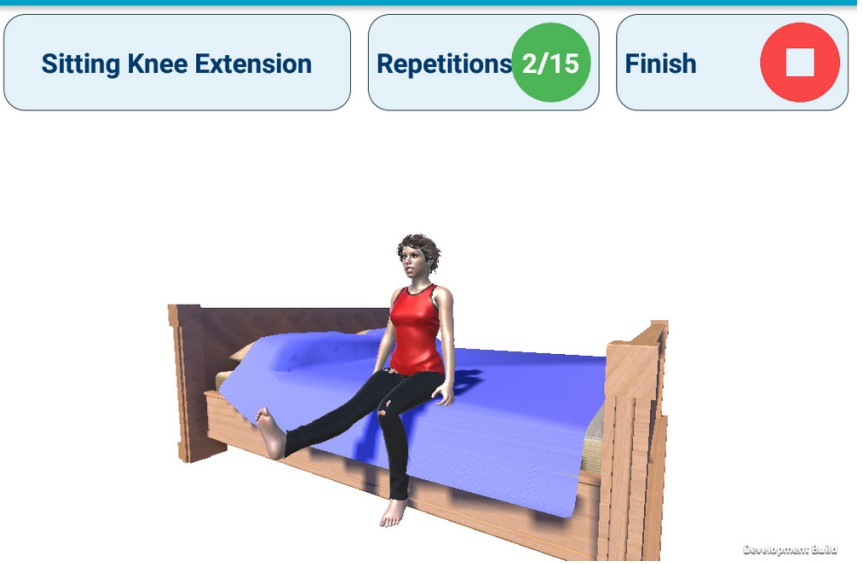

Figure 2 Screenshot of Android application during exercise mode.

testing on an Oxford scale it's a very subjective thing. (Physiotherapist)

Those participants working in the postacute phase of care stated that feedback was primarily offered verbally, and it is not possible to offer other feedback between clinic appointments.

I guess just verbal feedback is our only option... but when they go home we're not giving them any feedback 'till they come back to the clinic. (Physiotherapist)

\section{Perceptions and knowledge of wearables}

Participants were aware of wearable technology, with many stating they have used some sort of wearable device in their personal lives, yet discussion was almost exclusively on the application in the fitness space.

The apps on the smartphone that you have to have a phone in your pocket for the app to work and it tracks how many steps you've done. I know about Fitbit. (Clinical nurse specialist)

Participants also discussed the motivational aspect associated with using a wearable device. They felt that as well as motivating the user to meet their goals, the opportunity to track their own data and analyse their results was of benefit.

You kind of got little rewards or prompts from it you know, 'congratulations you've done 5 days in a row' and I liked that. (Occupational therapist)

Drawbacks of such technology were also highlighted. One participant felt that it would not be beneficial for them to use wearables themselves, with others reporting the amount of choice being overwhelming, and questioning the validity of such measures.

How can it differentiate between height and weight, and I don't know that is always taken into account 
with some of these technologies. (Clinical nurse specialist)

\section{Opportunities and challenges for connected health in orthopaedic rehabilitation}

Participants were unanimous in agreement that there is an opportunity and wide scope for connected health interventions, including wearable and mobile technology, to play a role in orthopaedic rehabilitation.

I think there's definitely a place for it, because it's so much more in people's lives than ever. Lots of people like apps now and they like to tag into something, and if it can give more specific individual feedback then there's definitely a role for it to be a part of their progression, especially resulting in something that will rehab for months. (Physiotherapist)

A number of potential features were highlighted that clinicians would consider useful, such as progress tracking, feedback, remote monitoring, regular range of motion measurement and counting repetitions.

Even something like a Fitbit that showed a graph that you can go and check if they've actually done their exercise and if they've achieved their targets. (Staff nurse)

I love the idea of real-time feedback, the idea something can tell you you're doing something wrong immediately and help you correct it. (Occupational therapist)

Participants were also quick to point out the challenges of delivering healthcare solutions in this way. All participants interviewed stated that the usability of any technology would be a significant barrier to engagement that will need to be overcome.

I mean if the interface is difficult or if something, if errors keep occurring. I think the more simple a thing is and the more intuitive it is, the easier it will be from an uptake and ongoing compliance issue. (Occupational therapist)

Compliance with use of any connected health intervention was widely reported to be a challenge.

I think there are an awful lot of opportunities there but a lot of it comes down to patient compliance. (Clinical nurse specialist)

There were additional challenges relating to the reliability and validity of any such technology, with discussion of aspects such as internet capability, bugs and glitches.

The technology actually working, how many times you know, the lack of Wi-Fi, computer crashing... I suppose they would be the huge elements. (Clinical nurse specialist)
Data security challenges were also noted, with the potential for confidential healthcare data being accessed without permission.

One would have said data protection, that's always going to be your main challenge. (Clinical nurse specialist)

Participants also spoke of the important balance in the patient-clinician relationship, and the need for technology to enhance the role of the professional, rather than attempt to replace the clinician. It was noted that such technologies need to work without shifting the focus away from the patient's own self-management.

So I would think with technology the person who has to be driving the connection is the patient. (Clinical nurse specialist)

The final notable subtheme to arise when discussing challenges of connected health systems, is the user's own ability and confidence with technology.

If you look at the elderly population that are primarily receiving joint replacements, are a lot of them au fait with? Some of them would be but presumably there are a large percentage who aren't au fait with iPads, iPhone and similar technologies. (Physiotherapist)

However, other participants offered counter arguments that this may not be the case compared with years gone by.

I suppose, a lot of people have the technology, that's not as much a barrier. It's so accessible now on smartphones and so many people have smartphones. I'm seeing 90year olds using Skype and Facebook and I think that barrier is reducing. (Occupational therapist)

\section{Perceived impact of exemplar system}

Having completed a demonstration of the prototype exercise biofeedback system as described above, all participants perceived this system as potentially having a positive impact in orthopaedic rehabilitation. Every participant noted the ability to capture and track data in a manner that has not previously been possible as a major benefit. Participants were excited by the prospect of tracking and leveraging metrics such as adherence, difficulty, pain and mood to guide their decisions.

Then at least you can track as well, to see if they're actually using it at all. Because if they're not using it then they're likely not doing anything. (Physiotherapist)

One of my favourite things about it is that you can score the difficulty, if somebody is scoring 5, 5, 5 then they're finding it too difficult but if they're scoring $1,1,1$ you can progress them on very quickly. (Physiotherapist)

They also felt using this system would improve the outcome of rehabilitation. They suggested that if the 
patient was demonstrating greater adherence, this would be of benefit.

It'll improve the quality of the patient's rehab. So I think they will be more likely to follow more structured programmes when they have the visual feedback to do it. (Physiotherapist)

I think they would rehab quicker and be more confident in their rehab, which is the best thing. (Staff nurse)

Through instilling added reassurance and motivation, clinicians felt that this system could improve the patient experience.

I think it will enhance patient confidence in their recovery... it's reassuring to know you're on track. (Clinical nurse specialist)

Participants suggested this system could contribute to a more efficient healthcare system, both for the patient and the service provider.

... you're hopefully not going to need MUA's (manipulation under anaesthetic) and that kind of thing because you're doing it right from the beginning. (Staff nurse)

As a patient, I would imagine that if I was doing my exercises correctly, I would need less appointment time with a physiotherapist... If I wasn't doing the exercises correctly, the physiotherapist might call me to go through how to do it, but if I'm doing my exercises correctly, if I'm reaching the goals I need to and the activity levels that I need to, why do I need to come in and see a physio? (Clinical nurse specialist)

\section{Challenges}

Participants also felt that this increased efficiency could emerge as a challenge however, as there is a risk of the patient placing a reliance on the technology over the human. Although there was an acknowledgement from some that this would not be the case.

As long as I follow it... I wouldn't go and see the physio again. (Orthopaedic assistant)

It's not going to take the place of the therapist you know, but it just serves to really hammer home the message you're trying to get across to the patient. (Physiotherapist)

A major challenge for such a system which was discussed by five clinicians, related to the accuracy of the data provided by the technology.

The worry that it won't be giving you the right information and then it's going to skew your thought process and that of others because you can only report on what you're being given. (Physiotherapist)

Interviews highlighted the difficulty of tailoring an automated programme to the individual, with only a small number of standard exercises included in this system compared with the breadth of options prescribed in clinical practice.

My issue would be if we use this app after two weeks should we not be progressing their exercises so you're no longer going to be performing those exercises. (Physiotherapist)

An interesting discussion evolved with a number of participants relating to what a system such as this would do for the responsibility of care, with some clinicians stating that they feel no amount of technology will promote self-management.

Some people would see that as a tool for assisting self-management, other people no matter how much education or technology you provide, will still see their recovery as incumbent on the healthcare professional looking after them. (Clinical nurse specialist)

One feature of the remote monitoring aspect of the prototype system is to send an alert to the clinician if there is a concerning change in the data, but some participants stated that the responsibility of the clinician would have to be clearly defined in this instance, in order not to detract from the patient's own self-management.

I guess it depends who is responsible for their care... I wouldn't want alerts about patients who went home a week ago but are in 10/10 pain. I want to educate that patient and put that responsibility on them, the more we give patients the responsibility the better. By doing that alert it makes the patient more passive and we want to encourage the patient to take responsibility, educate them on taking steps like icing and taking pain medication, and then if it still doesn't settle down, then the patient needs to call. I think if we put the responsibility onto us we're leading down a dangerous path. (Physiotherapist)

\section{DISCUSSION \\ Principal findings}

This study has found that clinicians see technology as having the potential to improve and assist the rehabilitation process, but there are numerous barriers to overcome when designing such solutions. Clinicians highlighted a need for a solution which deals with the issue of exercise adherence and lack of support for patients at home. ${ }^{45}$ When presented with a demonstration of the prototype system, all participants felt that this type of technology has the potential to positively impact on the outcomes of orthopaedic rehabilitation; however, there are challenges in ensuring the accuracy of information provided, and the ability to tailor such systems to the individual. There was strong interest in the opportunity to track data and maximise the effectiveness of rehabilitation, yet it was felt that such systems can undergo further iterations to extend this impact. 
Current clinical practice provides little opportunity for monitoring or feedback outside of the clinic. It has been shown that patients with negative experiences of TKR have unmet needs regarding support and managing expectations, ${ }^{21}$ yet these results suggest there is a heavy reliance on the patient's own self-motivation at home, with limited monitoring or feedback from clinicians. Participants also reported using a combination of objective and subjective outcome measures for monitoring, but these measures are only captured at limited timepoints and self-reported measures such as adherence are well reported to be unreliable. ${ }^{42}$ The results from this study would suggest there is benefit in capturing further objective outcome measures such as movement quality, or accurate monitoring of range of movement and adherence remotely, both of which are feasible with the use of IMUs. $^{323}$

There is growing evidence that clinicians see wearable devices supporting various aspects of assessment and intervention in rehabilitation, ${ }^{24}{ }^{25}$ and the results of this study further add to this evidence. Furthermore, these findings show that clinicians share the concerns reported in the literature relating to usability, data protection and reliability, which are known to be problematic with wearable devices. ${ }^{26-28}$ Many researchers are investigating the technical feasibility of using wearable sensing systems to support rehabilitation, ${ }^{29-32}$ but in a recent systematic review, few have conducted user evaluations to guide the design of such systems in a user-centred manner. ${ }^{33}$

\section{Prototype system evaluation}

Most clinicians believed the demonstrated prototype would improve the quality of patient rehabilitation by fostering greater motivation and self-efficacy, underpinned by the concept of persuasive technology. ${ }^{34}$ Clinicians were also excited by the opportunity to capture timely and objective data in a manner not currently available in practice. ${ }^{8}$ It was felt that this data-driven aspect could enhance clinical decision making, a perceived benefit that could increase usefulness and facilitate technology acceptance. ${ }^{36}$ It is perceived that this system could increase adherence to exercise programmes by using evidence-based interventions discussed in the literature to improve adherence in an automated manner. ${ }^{3}{ }^{37-39}$ Interestingly, several participants felt this system had the potential to improve the efficiency of the healthcare system, by harnessing the data collected to make better use of appointment scheduling, which has been highlighted as a design requirement by healthcare professionals previously. ${ }^{154}$ Existing literature has shown the ability of IMU-based systems to record and classify exercise technique, ${ }^{11} 18$ and this study has highlighted the clinical applications of such work.

\section{Challenges}

There were conflicting reports within these results as to whether such a system could lead to a reliance on technology rather than the clinician; however, previous research has suggested healthcare professionals see technology as having the ability to augment the clinician's decision-making process, rather than seek to replace the human expert. ${ }^{24}$ There was also concern that this system might increase the responsibility clinicians have for their patients by sending alerts, although this may be used to illustrate shortcomings in current practice. A clinician may consider a user educated in self-management and not wish for alerts, yet if this user continues to report severe symptoms, either the success of education is questionable or further assessment is required. When developing such systems, there is also a need to allow for customisation of exercise programmes from the clinician, with participants highlighting the variance between individuals, the need for a larger exercise library and the desire to choose when progressions should be offered. The major challenge in the development of such a support system which harnesses machine learning technology is in ensuring the accuracy and reliability of the information provided, as clinicians were concerned that inaccuracy could negatively impact user engagement. This study has shown the need for clear and transparent real-world validation, and end-user evaluation of any such system prior to implementation, an aspect that is lacking in the current literature. $^{2833}$

\section{Limitations}

Due to the qualitative nature of this study, there are number of factors to consider when reviewing this research. All participants were provided with a demonstration of the prototype system on one occasion only. It is quite possible that with ongoing use, or given further time to reflect on the system, the participant's opinions may change. It is important to highlight that these results are derived solely from the participants' own opinions via interviews, and in order to determine the objective clinical impact of such a system, further research needs to take place in the form of a longitudinal study assessing a variety of outcomes with a mixed-methods approach. This sample was selected from a single institution in the private healthcare sector, and while this sample comes from a cross-section of the multidisciplinary team, it does not guarantee that the opinions provided reflect the wider population of rehabilitation professionals. Finally, the purpose of this study was to assess clinician perceptions of wearable systems, nonetheless the lack of patient involvement is a limitation in this study. Despite these limitations, this paper provides evidence to support the need, ongoing design and development, and use of such systems in clinical practice.

\section{Future work}

Having now understood that there is a positive perceived impact for this system, future work will seek to deploy the platform as part of a pilot study with patients following TKR surgery. This work will seek to answer some of the questions posed by participants in this study relating to usability and engagement, while including the patient as well as the clinician in the design process to develop 
further iterations, prior to objective assessment of clinical impact and real-world validation.

\section{CONCLUSIONS}

Rehabilitation following joint replacement is complex and varied, yet there is a reliance on the patient's own self-management and motivation to maximise the effect of the home exercise programme, with little opportunity for expert monitoring, assessment or feedback outside of the clinic setting. A prototype system for interactive exercise biofeedback consisting of a single wearable sensor and an Android application was perceived to potentially have a positive impact on the rehabilitation of patients following knee replacement surgery. Clinicians were excited by the opportunity to continuously track data in real-time, and felt the exercise technique and adherence feedback would further support the patient in the home environment. However, there are challenges in the design and implementation of such systems to ensure acceptability. In order to achieve successful deployment, these technologies need to be evaluated by clinicians as well as patients as the end-users, alongside real-world validation of the technical aspects of any such platforms.

Acknowledgements The authors would like to thank Professor Tahar Kechadi for his assistance in the development of the prototype biofeedback system.

Contributors $\mathrm{RA}, \mathrm{PS}, \mathrm{AD}$ and $\mathrm{BC}$ conceived and designed the study. RA conducted the recruitment and data collection with the assistance of $P S, A D$ and $M N$. $A B$ contributed to the development of the prototype biofeedback system. RA and PS conducted the data analyses and drafted and revised the manuscript. All authors read and approved the final manuscript.

Funding This project forms part of the CHESS (Connected Health Early Stage Researcher Support System) Innovation Training Network and has received funding from the European Union's Horizon 2020 research and innovation programme under the Marie Sklodowska-Curie grant agreement no. 676201.

Competing interests None declared.

Patient consent Not required.

Ethics approval The research ethics committee of Beacon Hospital approved this study (REF: BEA0065).

Provenance and peer review Not commissioned; externally peer reviewed.

Data sharing statement № additional data available.

Open access This is an open access article distributed in accordance with the Creative Commons Attribution Non Commercial (CC BY-NC 4.0) license, which permits others to distribute, remix, adapt, build upon this work non-commercially, and license their derivative works on different terms, provided the original work is properly cited, appropriate credit is given, any changes made indicated, and the use is non-commercial. See: http://creativecommons.org/licenses/by-nc/4.0/.

\section{REFERENCES}

1. Maradit Kremers H, Larson DR, Crowson CS, et al. Prevalence of total hip and knee replacement in the United States. J Bone Joint Surg Am 2015;97:1386-97.

2. American Academy of Orthopaedic Surgeons, 2017. Total knee replacement exercise guide. http://orthoinfo.aaos.org/topic.cfm? topic=a00301 (Accessed 24 May 2018).

3. Argent R, Daly A, Caulfield B. Patient Involvement With Home-Based Exercise Programs: Can Connected Health Interventions Influence Adherence? JMIR Mhealth Uhealth 2018;6:e47.

4. Bassett SF. The assessment of patient adherence to physiotherapy rehabilitation. New Zeal J Physiother 2003;31:60-6.
5. Perry MA, Hudson HS, Meys S, et al. Older adults' experiences regarding discharge from hospital following orthopaedic intervention: a metasynthesis. Disabil Rehabil 2012;34:267-78.

6. Friedrich M, Cermak T, Maderbacher P. The effect of brochure use versus therapist teaching on patients performing therapeutic exercise and on changes in impairment status. Phys Ther 1996;76:1082-8.

7. Holden MA, Haywood KL, Potia TA, et al. Recommendations for exercise adherence measures in musculoskeletal settings: a systematic review and consensus meeting (protocol). Syst Rev 2014;3:10.

8. Caulfield BM, Donnelly SC. What is connected health and why will it change your practice? QJM 2013;106:703-7.

9. Burns A, Greene BR, McGrath MJ, et al. SHIMMERTM - A wireless sensor platform for noninvasive biomedical research. IEEE Sens J 2010;10:1527-34.

10. Giggins OM, Persson UM, Caulfield B. Biofeedback in rehabilitation. J Neuroeng Rehabil 2013;10:60.

11. Giggins OM, Sweeney KT, Caulfield B. Rehabilitation exercise assessment using inertial sensors: a cross-sectional analytical study. J Neuroeng Rehabil 2014;11:158.

12. O'Reilly MA, Whelan DF, Ward TE, et al. Technology in strength and conditioning: assessing bodyweight squat technique with wearable sensors. J Strength Cond Res 2017;31:2303-12.

13. Michie S, Yardley L, West R, et al. Developing and evaluating digital interventions to promote behavior change in health and health care: recommendations resulting from an international workshop. J Med Internet Res 2017;19:e232.

14. Shah SG, Robinson I. Benefits of and barriers to involving users in medical device technology development and evaluation. Int $J$ Technol Assess Health Care 2007;23:131-7.

15. Bergmann JH, McGregor AH. Body-worn sensor design: what do patients and clinicians want? Ann Biomed Eng 2011;39:2299-312.

16. Blumenthal J, Wilkinson A, Chignell M. Physiotherapists' and physiotherapy students' perspectives on the use of mobile or wearable technology in their practice. Physiother Can 2018;70:251-61.

17. Ritchie J. Qualitative research practice: a guide for social science students and researchers. 2nd edn. Los Angeles, CA: Sage Publications Sage CA, 2014.

18. Bevilacqua A, Huang B, Argent R, et al. Automatic Classification of Knee Rehabilitation Exercises Using a Single Inertial Sensor: a Case Study. 2018 IEEE 15th International conference on wearable and implantable Body Sensor Networks (BSN): IEEE, 2018:21-4.

19. Fereday J, Muir-Cochrane E. Demonstrating rigor using thematic analysis: a hybrid approach of inductive and deductive coding and theme development. Int J Qual Methods 2006;5:80-92.

20. Boeije H. A purposeful approach to the constant comparative method in the analysis of qualitative interviews. Quality and Quantity 2002;36:391-409.

21. Goldsmith LJ, Suryaprakash N, Randall E, et al. The importance of informational, clinical and personal support in patient experience with total knee replacement: a qualitative investigation. BMC Musculoskelet Disord 2017;18:1-13.

22. Hall AM, Kamper SJ, Hernon M, et al. Measurement tools for adherence to non-pharmacologic self-management treatment for chronic musculoskeletal conditions: a systematic review. Arch Phys Med Rehabil 2015;96:552-62.

23. Cooper G, Sheret I, McMillan L, et al. Inertial sensor-based knee flexion/extension angle estimation. J Biomech 2009;42:2678-85.

24. Papi E, Murtagh GM, McGregor AH. Wearable technologies in osteoarthritis: a qualitative study of clinicians' preferences. BMJ Open 2016;6:e009544

25. Sprint G, Cook DJ, Weeks DL. Designing wearable sensor-based analytics for quantitative mobility assessment. IEEE Int Conf Smart Comput SMARTCOMP 2016.

26. Piwek L, Ellis DA, Andrews $S$, et al. The rise of consumer health wearables: promises and barriers. PLoS Med 2016;13:e1001953.

27. Zapata BC, Fernández-Alemán JL, Idri A, et al. Empirical studies on usability of mHealth apps: a systematic literature review. J Med Syst 2015;39:1-19.

28. Peake JM, Kerr G, Sullivan JP. A critical review of consumer wearables, mobile applications, and equipment for providing biofeedback, monitoring stress, and sleep in physically active populations. Front Physiol 2018;9:743.

29. Ayoade M, Baillie L. A novel knee rehabilitation system for the home. Proceedings of the SIGCHI conference on human factors in computing systems: ACM, 2014:2521-30.

30. Chen KH, Chen PC, Liu KC, et al. Wearable sensor-based rehabilitation exercise assessment for knee osteoarthritis. Sensors 2015;15:4193-211. 
31. Brutovsky J, Novak D. Low-cost motivated rehabilitation system for post-operation exercises. Conf Proc IEEE Eng Med Biol Soc 2006:6663-6.

32. Kos A, Tomažič S, Umek A. Suitability of smartphone inertial sensors for real-time biofeedback applications. Sensors 2016;16:301.

33. O'Reilly M, Caulfield B, Ward T, et al. Wearable inertial sensor systems for lower limb exercise detection and evaluation: a systematic review. Sports Med 2018;48:1221-46.

34. Fogg BJ. Persuasive technology: using computers to change what we think and do. San Francisco, CA: Morgan Kaufmann, 2003.

35. Chow Y-W, Susilo W, Phillips JG, et al. Video games and virtual reality as persuasive technologies for health care: an overview.
J Wirel Mob Networks, Ubiquitous Comput Dependable Appl 2017:8:18-35.

36. Holden RJ, Karsh BT. The technology acceptance model: its past and its future in health care. J Biomed Inform 2010;43:159-72.

37. Jack K, McLean SM, Moffett JK, et al. Barriers to treatment adherence in physiotherapy outpatient clinics: a systematic review. Man Ther 2010;15:220-8.

38. Jordan JL, Holden MA, Mason EE, et al. Interventions to improve adherence to exercise for chronic musculoskeletal pain in adults. Cochrane Database Syst Rev 2010;1:CD005956.

39. Peek K, Sanson-Fisher R, Mackenzie L, et al. Interventions to aid patient adherence to physiotherapist prescribed self-management strategies: a systematic review. Physiotherapy 2016;102:127-35. 